\title{
Rice seeds identification based on back propagation neural network model
}

\author{
Xuebin Feng, Peijun He, Huaxi Zhang, Wenqing Yin, Yan Qian, Peng Cao, Fei Hu* \\ (College of Engineering, Nanjing Agricultural University, Nanjing 210031, China)
}

\begin{abstract}
Rice quality directly affects the final rice yield. In order to achieve rapid, non-destructive testing of rice seeds, this paper combines the three-dimensional laser scanning technology and back propagation (BP) neural network algorithm to build a rice seeds identification platform. The information on rice seed surface is collected from four angles and processed using Geomagic Studio software. Based on the noise filtering, smoothing of the point cloud, vulnerability repair, and downsampling, the three-dimensional (3D) morphological characteristics of a rice seed surface, and the projection features of the main plane cross-section are obtained through the calculation of the features. The experiments were performed on five rice varieties, including Da Hua aromatic glutinous, Hong Shi I, Tian You VIII, Xin Dao X, and Yu Jing VI. The resulting input vector consisted respectively of: (1) nine 3D morphological surface features, (2) nine projection features of the main cross-section plane of rice, and (3) all of the above features. The results showed that for an input vector consisting of nine surface 3D morphological features, the recognition rate of the five rice varieties was $95 \%, 96 \%, 87 \%, 93 \%$, and $89 \%$, respectively; for an input vector consisting of nine projection features of the main cross-section plane of rice seeds, the recognition rate was $96 \%$, $96 \%, 90 \%, 92 \%$, and $89 \%$, respectively; and lastly, for an input vector consisting of all the features, the highest recognition rate of $96 \%, 97 \%, 91 \%, 94 \%$, and $90 \%$, respectively, was achieved. The analysis showed that rice varieties could be identified by using 3D laser scanning. Therefore, the proposed method can improve the accuracy of rice varieties identification.
\end{abstract}

Keywords: rice seeds identification, BP neural network, three-dimensional laser scanning, features, point cloud DOI: $10.25165 /$ j.ijabe.20191206.5044

Citation: Feng X B, He P J, Zhang H X, Yin W Q, Qian Y, Cao P, et al. Rice seeds identification based on back propagation neural network model. Int J Agric \& Biol Eng, 2019; 12(6): 122-128.

\section{Introduction}

Rice is not only the main food crop in the agricultural production in China but also the staple food of about half of the world population. In the process of rice cultivation, the excellent rice varieties tend to increase agricultural production efficiently, but in fact, high-quality rice is often mixed with poor-quality rice, resulting in purity falling of rice varieties, which further affects the overall yield $^{[1]}$. Therefore, to ensure a stable and high yield of high-quality rice, it is necessary to detect the purity of rice seeds before rice sowing.

In recent years, due to the development of semiconductors and microelectronics, the advanced electronic equipment and the new technology of surveying and mapping have been continuously improving. The three-dimensional (3D) laser scanning technology has merged the optics, mechanics, electronics, and other technologies, thus improving the traditional measurement technologies, providing a wide-range, high-efficiency, high-precision 3D information acquisition ${ }^{[2]}$, and enabling the scanning of object optical characteristics.

\section{Received date: 2019-03-26 Accepted date: 2019-08-28}

Biographies: Xuebin Feng, PhD, Lecturer, research interest: agricultural engineering, Email: fengxuebin6@163.com; Peijun He, Bachelor, research interest: agricultural engineering, Email: 30316416@njau.edu.cn; Huaxi Zhang, Master, research interest: agricultural engineering, Email: cczhanghuaxi@ 163.com; Wenqing Yin, PhD, Professor, research interest: agricultural engineering, Email: yinwq@njau.edu.cn; Yan Qian, PhD, Associate Professor, research interest: agricultural engineering, Email: qianyan@njau.edu.cn; Peng Cao, Master, research interest: agricultural engineering, Email: 15189838054@ 163.com.

*Corresponding author: Fei Hu, PhD, Associate Professor, research interest: agricultural engineering, College of Engineering, Nanjing Agricultural University, Nanjing 210031, China. Email: hufeinjau@163.com.
The detailed information on a $3 \mathrm{D}$ rice surface collected in an indirect way without damaging the rice denotes the reliable initial data for further rise seed characteristics determination. Moreover, the characteristic parameters have a space shift rotation invariance, so they can greatly enhance the identification accuracy of rice varieties.

The BP neural network is a hierarchical feedforward neural network. Its input layer receives input signal, which is then processed and transmitted to the hidden layer, and after that, transmitted to the output layer. Due to the strong ability of nonlinear mapping, self-learning, adaptive capacity, generalization ability, and fault tolerance, the BP neural networks are the most widely used neural network models in face recognition ${ }^{[3,4]}$, license plate recognition ${ }^{[5]}$, palmprint recognition ${ }^{[6]}$, etc. In the agricultural field, the BP neural networks are used for identification of cotton diseases ${ }^{[7]}$, transgenic soybean ${ }^{[8]}$, and corn seeds ${ }^{[9]}$.

Tao and $\mathrm{Zhou}^{[10]}$ proposed an automatic segmentation and recognition method for the robot based apple picking using the point cloud data, where the color features and 3D geometry features were fused, which achieved the classification accuracy of $92.30 \%$. For the identification of large objects, such as apples, features can be obtained easily, and the error influence on final results is not obvious. However, due to the high precision requirements, for small objects, such as rice seeds, the error caused by common measurement methods is enormous, and the recognition efficiency is generally hard to guarantee. Shantaiya and Ansari ${ }^{[11]}$ used a color digital camera to collect the sample images of rice seeds and obtained nine morphological characteristics, and six color features were obtained. Six kinds of rice seeds were identified with the average classification accuracy of $84.8 \%$, which proved that rice seeds could be successfully identified by an artificial neural network.

In summary, the detailed 3D contour information can be 
collected efficiently by using a 3D laser scanning, and a BP neural network can be employed to fit the data characteristics. In our previous work, our research team proposed a calculation method of surface shape features of rice seeds based on the point cloud, obtaining nine $3 \mathrm{D}$ features of rice seeds ${ }^{[12]}$. Based on previous research, nine projection features of the main cross-section plane of rice seeds are obtained, and 18 features of rice seeds can be obtained in total. In this study, a laser scanning is combined with a BP algorithm, and nine 3D morphological surface features, nine projection features of the main cross-section plane of rice seeds, and all 18 features are input to the BP neural network, respectively, with an aim to get an ideal recognition rate of rice seed varieties.

\section{Materials and methods}

The objective of this work is to identify the rice seeds by using 3D laser scanning technology. Based on a BP model, the calculation method of 3D morphological characteristics of rice seed surface and the projection features of the main plane cross-section of rice seeds are presented. The identification of rice seed varieties is performed by an artificial neural network model; the effect of characteristic parameters on the classification and identification performance of rice seeds is studied, and then used as a theoretical basis for classification and identification of rice seed varieties. The flowchart of the proposed method is shown in Figure 1.

\begin{tabular}{|c|}
\hline Build 3D laser scanning system \\
\hline $\begin{array}{c}\text { Collect surface point cloud data of } \\
\text { rice and preprocess the collected data }\end{array}$ \\
\hline $\begin{array}{c}\text { Calculate 3D morphological } \\
\text { characteristics of rice surface }\end{array}$ \\
\hline $\begin{array}{c}\text { Calculate projection feature of } \\
\text { main plane cross-section of rice }\end{array}$ \\
\hline \\
Build BP neural network \\
\hline $\begin{array}{c}\text { Substitute the resulting features } \\
\text { into the BP neural network }\end{array}$ \\
\hline
\end{tabular}

Figure 1 Flowchart of the rice seeds identification method

\subsection{Rice seed varieties}

In this work, five varieties of rice seeds, including Da Hua aromatic glutinous, Hong Shi I, Tian You VIII, Xin Dao X, and Yu Jing VI, are used for method development, and 50 samples of each variety are used for method testing. Some of the samples used in the test are presented in Figure 2.

\section{0 aroma Hua 1) 1) 0100010000 D010001) Hong Shi I 00000000000000000 Tian You VIII 1000000000001000 xin Dao $\mathrm{x}$ 00000000000000000 Yu Jing VI}

Figure 2 Rice seeds samples used in the test

\subsection{Three-dimensional laser scanning system}

Here, we use a 3D line laser scanner (model SED, Beijing Winner Optical Instruments Co., Ltd, Beijing, China) with a maximum scanning accuracy of $0.01 \mathrm{~mm}$, as shown in Figure $3 \mathrm{a}$.
The measuring head of the scanner is installed on the mechanical platform by a transfer plate, which is driven by a servo motor. The maximum moving distance of a measuring head along each axis is $400 \mathrm{~mm}$, and the positioning accuracy is $0.1 \mathrm{um}$. After system initialization, using the movement control cards and image acquisition card driver measuring head, the digital scanning process of a rice seed surface is performed, and the measured point cloud data are stored in the form of a text file on the computer for further analysis.

The 3D laser scanning system is composed of the control module, drive module, and measurement module. The control module includes the computer, image acquisition card, and motion control card embedded in a computer motherboard. The driving module consists of the three-axis servo actuators and servo motors. The measuring module mainly refers to the measuring head, which includes two CCD lens and laser generator, as shown in Figure $3 b$.
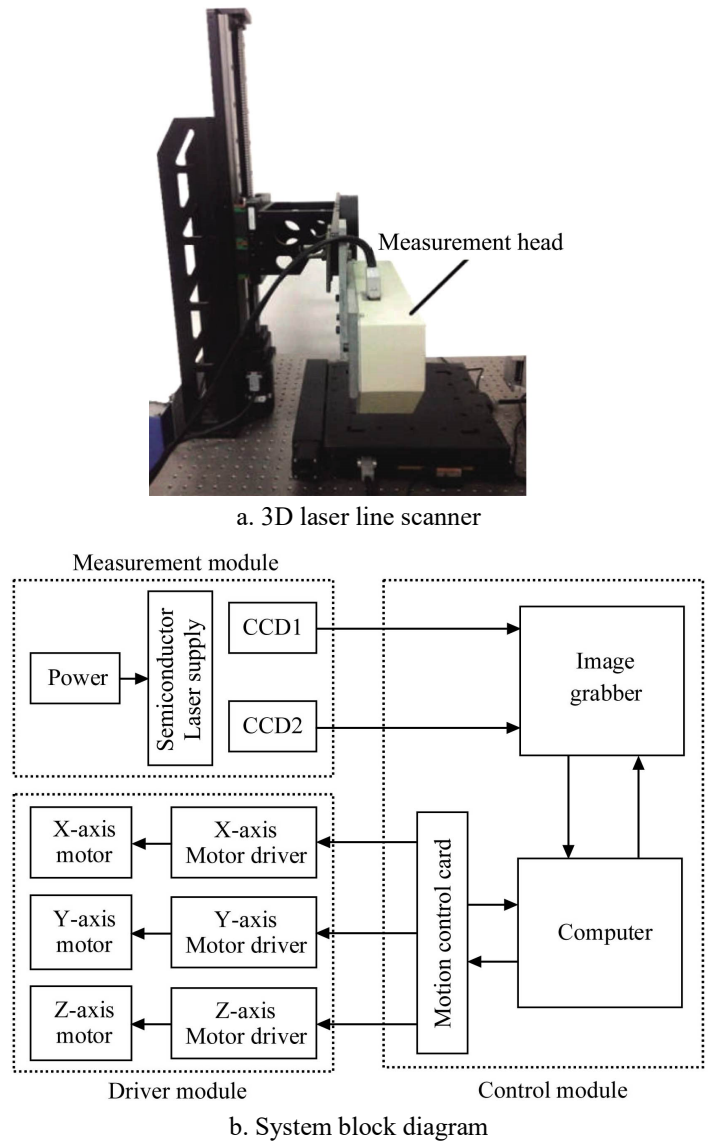

Figure 3 3D laser scanning system

\subsection{Extraction of rice seed features}

In order to characterize the point cloud characteristics of different rice seed varieties, and classify the varieties accordingly, 18 features of rice seeds are extracted using the 3D point cloud model of rice seeds. The flowchart of the point cloud data processing is shown in Figure 4.

2.3.1 Collection and preprocessing of rice seed surface point cloud data

Collection of the point cloud data is the primary step of the calculation of 3D morphological characteristics of rice seeds, and also the basis for classification and identification of rice seed varieties. The data on rice seed surfaces are collected from four perspectives, which is consistent with the procedure of the previous research adopted by our team ${ }^{[12]}$, the registration is performed using Geomagic Studio software (2013, Geomagic, North Carolina, America). 


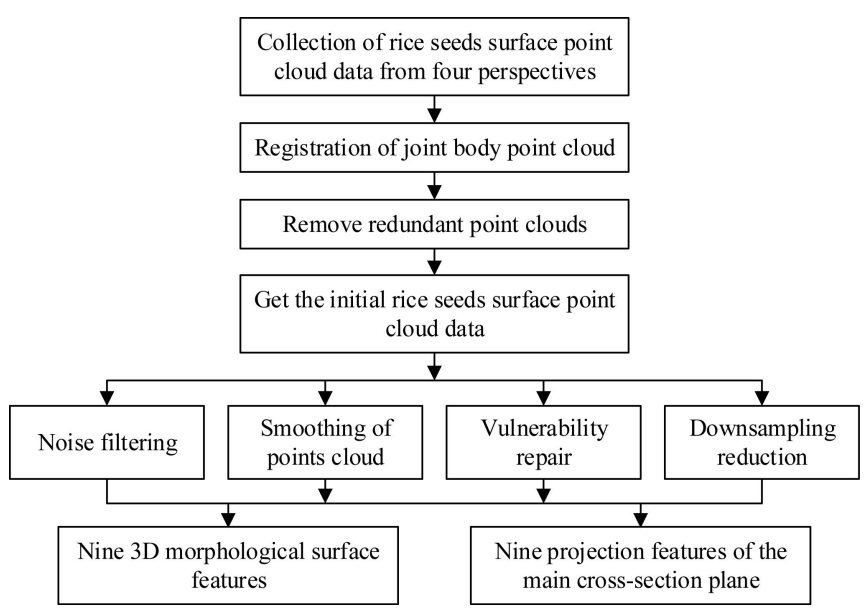

Figure 4 Data processing flowchart

Due to the noise, voids, and data redundancy of the acquired rice seeds point cloud data, a basic preprocessing of the point cloud data is performed before the feature calculation, including the noise filtering, smoothing of points cloud, vulnerability repair, and downsampling reduction.

2.3.2 Calculation of three-dimensional morphology features of rice seed surface

(1) Computation of length, width, and thickness

An oriented bounding box (OBB) model, which represents the closest cuboid model of the point cloud model of rice seed, is constructed for computing the length, width, and thickness of the point cloud model. The length, width, and height of the OBB model represent the length, width, and thickness of the point cloud model, respectively. After the model coordinate system calibration, the three dimensions of the model coincide with the three axes of the coordinate system. We first find the maximum and minimum value on all the three axes and label them as $x_{\max }$, $x_{\min }, y_{\max }, y_{\min }, z_{\max }$, and $z_{\min }$, respectively. Then, the length $(L)$, width $(W)$, and height $(H)$ are calculated by:

$$
\begin{gathered}
L=x_{\max }-y_{\text {min }} \\
W=y_{\max }-y_{\text {min }} \\
H=z_{\max }-z_{\text {min }}
\end{gathered}
$$

(2) Calculation of rice seeds volume

Based on the surface morphology of rice seeds, a simplified calculation of rice seeds volume based on the point cloud model is proposed in this paper. The specific process is as follows.

a) Point cloud model cut

The point cloud model is divided along the $x$-axis at equal intervals to find the section plane closest to each point in the model, and calculate the projections of each point on the corresponding section plane. Thus, the point cloud model consisting of $n$ sections labeled as $P_{i}$, where $i=1,2, \ldots, n$, is obtained. In Figure 5 , a cross-section point cloud model is presented in the $y \mathrm{O} z$ plane. In Figure 5, it can be seen that the point cloud denotes a two-dimensional concave set of points.

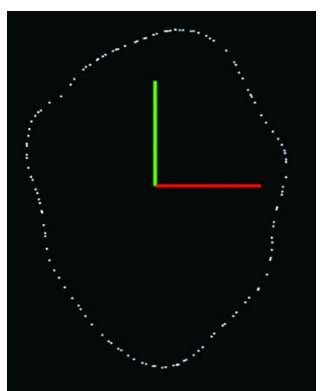

Figure 5 The section point cloud model b) Calculation of cross-section area

Suppose that the projection point cloud model is a set of concave-area points $U_{\mathrm{i}}$, which includes points $A\left(x_{1}, y_{1}\right), B\left(x_{2}, y_{2}\right)$, $C\left(x_{3}, y_{3}\right), D\left(x_{4}, y_{4}\right), E\left(x_{5}, y_{5}\right), F\left(x_{6}, y_{6}\right)$, and $G\left(x_{7}, y_{7}\right)$, while point $P\left(x_{\mathrm{p}}, y_{\mathrm{p}}\right)$ is outside the concave area, as shown in Figure 6. The concave area is constituted by a triangular vector area composed of the vertices of $P$ and $A-G$. When the clockwise direction is specified as a positive direction, the areas of $\triangle P A B, \triangle P B C$, and $\triangle P C D$ are all positive, and the areas of $\triangle P D E, \triangle P E F, \triangle P F G$, and $\triangle P G A$ are all negative.

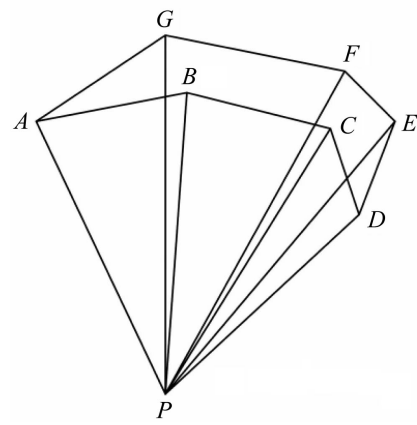

Figure 6 Schematic diagram of a concave area

Assume that $d_{A P}, d_{B P}$, and $d_{A B}$ represent the lengths of triangular sides $l_{A P}, l_{B P}$, and $l_{A B}$, respectively. Then, according to Heron's formula, it can be written that:

$$
S_{\triangle P A B}=\sqrt{\frac{l}{2}\left(\frac{l}{2}-d_{A P}\right)\left(\frac{l}{2}-d_{B P}\right)\left(\frac{l}{2}-d_{A B}\right)}
$$

By the analogy, the areas of $\triangle P B C, \triangle P C D, \triangle P D E, \triangle P E F$, $\triangle P F G$, and $\triangle P G A$ are $S_{\triangle P B C}, S_{\triangle P C D}, S_{\triangle P D E}, S_{\triangle P E F}, S_{\triangle P F G}$, and $S_{\triangle P G A}$, respectively. Then, it can be written:

$$
S=\left|S_{\triangle P A B}+S_{\triangle P B C}+S_{\triangle P C D}-S_{\triangle P D E}-S_{\triangle P E F}-S_{\triangle P F G}-S_{\triangle P G A}\right|
$$

where, $S$ represents the area of concave-area points $U_{i}$, and it is the same as the area of the projection point cloud model.

c) Calculation of point cloud model volume

Assume that the sectional areas of the model divided by the equidistant plane are $S_{0}, S_{1}, \ldots, S_{n}$. When the value of $n$ is large enough, the adjacent section cutting planes are approximately equal. Then, the volume of the point cloud model can be approximately calculated by:

$$
V \approx \frac{x_{\max }-x_{\min }}{n} \sum_{i=0}^{n-1} \frac{S_{i}+S_{i+1}}{2}
$$

And the $i^{\text {th }}$ round volume is given by:

$$
V_{i} \approx \frac{x_{\max }-x_{\min }}{n} \frac{S_{i}+S_{i+1}}{2}
$$

Assume the volume calculated in the $m^{\text {th }}$ iteration is $V_{m}$, and the absolute value of the difference between it and volume $V_{m-1}$ obtained in the previous iteration is less than a given threshold; then, it can be considered that the calculation result reached the actual accuracy; the model volume is $V_{m}$; otherwise, the next round of segmentation is performed on the point cloud model.

(3) Calculation of rice seed surface area

Surface area is one of the most important shape features of an object. The surface point cloud model is obtained by laser scanning technology. The surface area of rice seeds is calculated by establishing a triangular mesh of the rice seed point cloud model $^{[13,14]}$. The specific process is as follows.

a) First, a triangular mesh of the rice-seeds point cloud model is established. Then, a greedy projection algorithm ${ }^{[15]}$ is used for triangulation of the rice seeds point cloud. 
b) Then follows the calculation of the surface area of a triangle model. The given rice seeds triangular mesh model is made up of triangular patches $T=\left\{T_{1}, T_{2}, \ldots, T_{n}\right\}$, where $n$ is the number of triangular patches, $T_{i}$ is the $i^{\text {th }}$ triangular patch whose three vertices are $A_{i}, B_{i}$, and $C_{i}$. The corresponding triangle lengths $a_{i}, b_{i}$, and $c_{i}$ are calculated by the Euclidean distance formula, and the surface area of the triangular $S_{A i B i C i}$ can be calculated by Eq. (4) and Eq. (5). Then, the surface area of rice seeds is given by:

$$
S=\sum_{i=1}^{n} S_{A_{i} B_{i} C_{i}}
$$

Further, the needle degree, flatness, shape factor, and sphericity of rice seeds are calculated. The surface of rice seeds is approximately ellipsoid. Through the research on the outline shape of rice seeds, the needle index $\Theta$, flatness $\Phi$, shape factor $F$, and sphericity $\Psi$ are introduced as the quantitative indexes of rice seeds morphology ${ }^{[16]}$ to evaluate the contour characteristics of rice seeds, and they are respectively calculated by:

$$
\begin{aligned}
\Theta & =\frac{L}{W} \\
\Phi & =\frac{H}{W} \\
F & =\frac{H L}{W^{2}} \\
\psi & =\left(\frac{W H}{L^{2}}\right)^{\frac{1}{3}}
\end{aligned}
$$

2.3.3 Calculation of projection features of the main plane cross-section of rice seeds

The edge outline of rice seeds point model is extracted by a seed point outline extraction based on the Alpha Shape ${ }^{[17]}$, and the edge outline information is further optimized by the cubic spline interpolation ${ }^{[18]}$ to make the image clearer.

(1) Area. The six-view projection method of the orthogonal projection is used, and the resulting six-view areas are compared to get the largest area, which is labelled as $S_{x}$.

(2) Perimeter. The perimeter is calculated by using the line connecting the outline points and accumulating the distance between all the neighboring outline points, labeled as $C$.

(3) Length, width, and length-width ratio. The length of the projected rice-seeds point cloud model denotes the maximum distance between the two endpoints on the edge outline of the rice seeds, while the width is defined by the intersection of the vertical line passing through the midpoint of the long axis and rice seed edges. The length-width ratio, denoted as $C_{L W}$, is the ratio of length $L_{x}$ to width $W_{x}$.

Suppose the coordinates of the leftmost and rightmost points are $\left(x_{1}, y_{1}\right)$ and $\left(x_{2}, y_{2}\right)$, respectively; then, the distance between two points is the length of a rice seed $L_{x}$, and the outline of the long axis of the midpoint coordinates $\left(x_{m}, y_{m}\right)$ is:

$$
\begin{aligned}
& x_{m}=\frac{x_{1}+x_{2}}{2} \\
& y_{m}=\frac{y_{1}+y_{2}}{2}
\end{aligned}
$$

The slope $K$ of the long axis is defined by:

$$
K=\frac{y_{2}-y_{1}}{x_{2}-x_{1}}
$$

Then, the short axis at $K \neq 0$ is defined by:

$$
y=-\frac{1}{K}\left(x-x_{m}\right)+y_{m}=-\frac{x_{2}-x_{1}}{y_{2}-y_{1}}\left(x-x_{m}\right)+y_{m}
$$

The long axis is defined as the $x$-axis, while the short axis is defined as the $y$-axis. The contour points close to the $y$-axis in intervals $x>0$ and $x<0$ are determined respectively, which are also the two endpoints of the short axis. Then, the outline-width $W_{x}$ is calculated.

When $K=0$, the $\mathrm{x}$-axis coincides with the long axis, so the equation of the short axis of the contour is expressed as $x=0$. Similarly, the contour width $W_{x}$ can be obtained. The length-width ratio of the rice seeds is given by:

$$
C_{L W}=\frac{L_{x}}{W_{x}}
$$

(4) Maximum radius $\left(R_{M A}\right)$ and minimum radius $\left(R_{M I}\right)$. The maximum distance and the minimum distance of the center of the mass $c$ to the boundary contour denote the maximum radius and the minimum radius, respectively, and the radius ratio $C_{R}$ is the ratio of the maximum radius to the minimum radius; they are all very important geometric features that identify the morphology of the rice seeds. The center of mass of the rice seeds cloud point model is defined by:

$$
c=\frac{\sum_{i=1}^{N} p_{i}}{N}
$$

where, $p_{\mathrm{i}}$ is the edge contour of the rice seeds, and $N$ is the number of contour points on the edge of the rice seeds. Then, we calculate the distance $d$ between the center of the mass $c$ and each boundary point of the rice seeds, and then find their maximum value $d_{\max }$ and minimum value $d_{\min }$ to get the maximum and minimum radius of rice seeds, respectively. The radius ratio of the rice seeds is given by:

$$
C_{R}=\frac{R_{M A}}{R_{M I}}
$$

(5) Circularity. Circularity ${ }^{[19]}$ describes the compactness of a region to a certain degree; it is dimensionless and insensitive to the region size, and its mathematical expression is:

$$
\gamma=\frac{C^{2}}{4 \pi S_{x}}
$$

where, $C$ and $S_{x}$ are the perimeter and area of the projection cloud model of rice seeds, respectively.

\subsection{Classification and identification of rice seeds using BP neural network}

The BP neural network, which is also called the error reverse transmission neural network, is a neural network that uses the feedback values to adjust the connection weights between neurons continuously. In this work, a three-layer BP neural network that is trained with eighteen features of the rice seeds is used to evaluate the relationship between the combination of features and rice seed varieties. The specific steps are as follows.

(1) Training and test samples

Five varieties of rice seeds, namely Da Hua, Xiang Nuo, Hong Shi I, Tian You VIII, Xin Dao X, and Yu Jing VI, were selected as test samples, and there were 190 samples per each variety (950 samples in total). Further, 160 samples of every variety were randomly selected to train the network, and the other 30 samples were used to test the trained network model.

(2) Three-dimensional morphological features

Nine 3D morphological features of surfaces of the rice seeds, namely the length $L$, width $W$, thickness $H$, volume $V$, surface area $S$, needle degree $\Theta$, flatness $\Phi$, shape factor $F$, and sphericity $\Psi$; nine projection features of the main plane cross-section, namely the circumference $C$, area $S_{x}$, length $L_{x}$, width $W_{x}$, aspect ratio $C_{L W}$, 
maximum radius $R_{M A}$, minimum radius $R_{M I}$, radius ratio $C_{R}$, and circularity $\gamma$ were calculated.

(3) Hidden layer

The hidden layer of the BP neural network is crucial for overall network performance. The number of neurons in the hidden layer was selected according to $\sqrt{n_{1} * n_{2}}$, where $n_{1}$ denotes the number of input parameters, and $n_{2}$ denotes the number of output parameters; therefore, $n_{1}$ was defined as the input-vector dimension, and it was set to 9,9 , and 18 , respectively, while $n_{2}$ was defined as the output-vector dimension, and it was set to 5 .

\section{Results and discussion}

\subsection{Collection and preprocessing of point cloud data of rice} seed surface

As can be seen in the marked areas in Figure 7, the noise points on the rice seed surface were filtered, the missing parts were improved, and a few points represented the original morphological characteristics of the rice seeds. Thus, the preprocessing of the collected point cloud data of the rice seeds effectively solved the problems of noise points, voids, and data redundancy, obtaining more precise and smooth point cloud data.

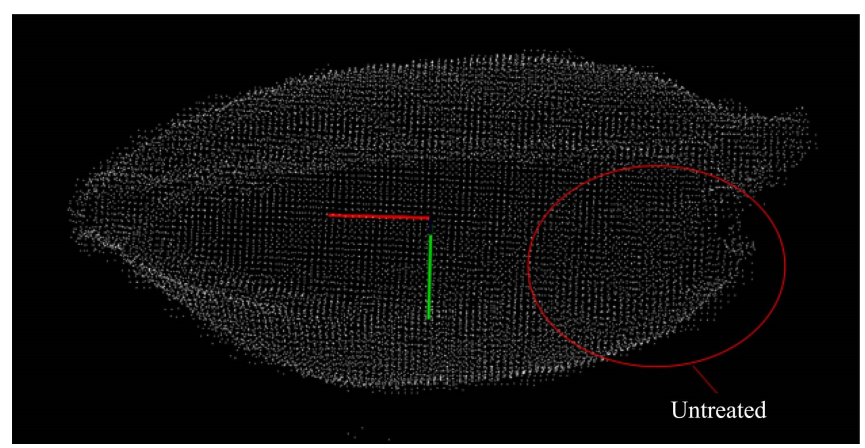

a. Untreated point cloud

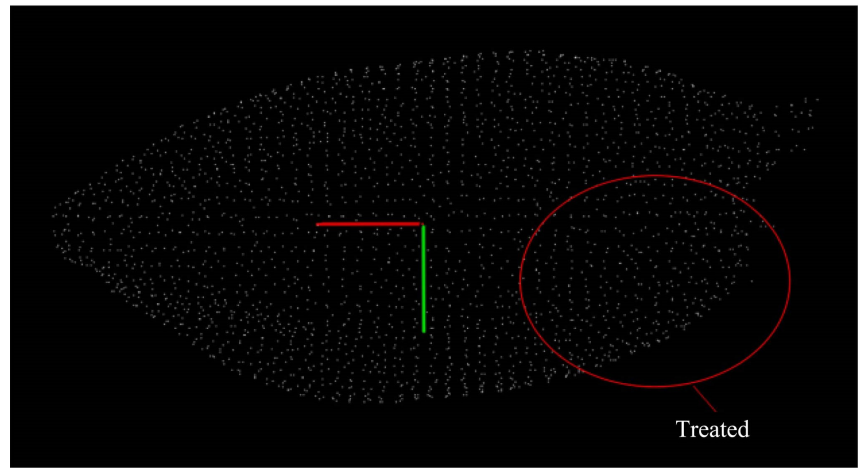

b. Treated point cloud

Figure 7 Point cloud data collection and preprocessing

\subsection{Experimental results}

3.2.1 Results of three-dimensional features of rice seed surface

We collected the rice-seed surface point cloud, and calculated the mean and standard deviation of 18 features; the results of the three-dimensional features of the rice seed surface are given in Table 1. Among nine morphological parameters, the length, width, thickness, volume, and surface area denoted the geometric parameters of rice seed surface, while the needle degree, flatness, shape factor, and sphericity denoted the synthetic indexes used to evaluate the rice seeds morphology. Taking rice seeds and pellets as test objects, in the previous study ${ }^{[12]}$, the calculated nine $3 \mathrm{D}$ morphological features of rice seeds were compared with the artificial measurements, and the relative error of less than 3\% was obtained, which verified the reliability of the data, and indicated that it could be used as the feature set of the BP neural networkbased identification and classification of rice seed varieties.

3.2.2 Projection features of the main plane cross-section of rice seeds

The projection features of the main plane cross-section of rice seeds were calculated by the projection point cloud model, and the obtained values are given in Table 2. Among nine morphological parameters, the length, width, perimeter, area, maximum radius, and minimum radius denoted the rice seeds shape characteristics. The length-width ratio, radius ratio, and circularity were calculated from the above six morphological characteristics. As can be seen in Table 2, the extracted parameters had the characteristics of differentiability and independence. These characteristics could be used as the feature set of the BP neural network-based identification and classification of rice seed varieties.

\subsubsection{Results for three different input vectors}

The results for three input vectors consisted of nine 3D morphological features, nine projection features of the main plane cross-section of rice seeds, and all the features, respectively, are shown in Figure 8.

For the first input vector, the number of neurons in the input layer was set to 9 , the number of neurons in the output layer was set to 5 , and the number of neurons in the hidden layer was set to 7 . The corresponding results are labeled as A in Figure 8. In Figure 8 , it can be seen that recognition rate of Hong Shi I was the highest, up to $96 \%$; the recognition rate of Tian You VIII was $87 \%$, which was the lowest recognition rate among the five varieties in the classification test. The average recognition rate was $92 \%$.

For the second input vector, the number of neurons in the input, hidden, and output layers were set to the same values as for the first input vector. As it can be seen in Figure 8, where the corresponding results are labeled as $\mathrm{B}$, the recognition rate of Hong Shi I and Da Hua aromatic glutinous was the highest, up to $96 \%$. The recognition rate of Yu Jing VI was $89 \%$, and that was the lowest recognition rate among the five varieties in the classification test. The average recognition rate was $92.6 \%$. Therefore, the recognition rate of Tian You VIII was improved by using nine morphological parameters as an input vector.

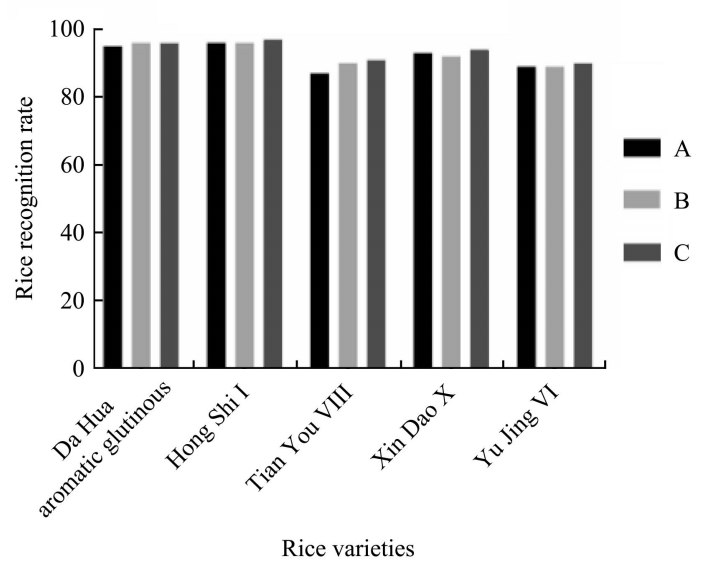

Figure 8 The results for three different input vectors

For the third input vector, there were 18 input parameters, so the number of neurons in the input layer was set to 18 , the number of neurons in the output layer was set to 5 , and the number of neurons in the hidden layer was set to 9. As can be seen in Figure 8 (refer to C), the recognition rate of Hong Shi I was the highest, up to $97 \%$. The recognition rate of Yu Jing VI was $90 \%$, and that was the lowest recognition rate among the five varieties in the classification test. The average recognition rate was $94 \%$. 
Table 1 Three-dimensional surface features of rice seeds

\begin{tabular}{|c|c|c|c|c|c|c|c|c|c|c|}
\hline \multirow{3}{*}{ Feature } & \multicolumn{10}{|c|}{ Rice variety } \\
\hline & \multicolumn{2}{|c|}{ Da Hua aromatic glutinous } & \multicolumn{2}{|c|}{ Hong Shi I } & \multicolumn{2}{|c|}{ Tian You VIII } & \multicolumn{2}{|c|}{ Xin Dao X } & \multicolumn{2}{|c|}{ Yu Jing VI } \\
\hline & Mean & SD & Mean & SD & Mean & SD & Mean & SD & Mean & SD \\
\hline Length $/ \mathrm{mm}$ & 7.05 & 0.15 & 8.02 & 0.10 & 7.49 & 0.13 & 7.05 & 0.09 & 7.55 & 0.09 \\
\hline Width/mm & 3.75 & 0.09 & 3.34 & 0.09 & 3.40 & 0.03 & 3.16 & 0.09 & 3.26 & 0.07 \\
\hline Length-width ratio/mm & 2.51 & 0.10 & 2.48 & 0.06 & 2.4 & 0.05 & 2.34 & 0.09 & 2.4 & 0.03 \\
\hline Perimeter $/ \mathrm{mm}$ & 27.33 & 1.92 & 27.63 & 1.10 & 25.06 & 0.53 & 22.72 & 1.50 & 24.43 & 0.87 \\
\hline Area $/ \mathrm{mm}^{2}$ & 51.92 & 2.11 & 54.28 & 1.63 & 49.15 & 0.81 & 45.38 & 1.51 & 48.25 & 1.11 \\
\hline The maximum radius $/ \mathrm{mm}$ & 1.88 & 0.05 & 2.40 & 0.07 & 2.20 & 0.04 & 2.24 & 0.06 & 2.32 & 0.06 \\
\hline The minimum radius $/ \mathrm{mm}$ & 0.67 & 0.03 & 0.74 & 0.03 & 0.71 & 0.02 & 0.74 & 0.03 & 0.74 & 0.02 \\
\hline Radius ratio & 1.26 & 0.08 & 1.78 & 0.11 & 1.56 & 0.05 & 1.66 & 0.10 & 1.71 & 0.08 \\
\hline Circularity & 0.57 & 0.01 & 0.51 & 0.01 & 0.53 & 0.01 & 0.53 & 0.01 & 0.52 & 0.01 \\
\hline
\end{tabular}

Note: SD is the standard deviation.

Table 2 Projection features of the main plane cross-section of rice seeds

\begin{tabular}{|c|c|c|c|c|c|c|c|c|c|c|}
\hline \multirow{3}{*}{ Feature } & \multicolumn{10}{|c|}{ Rice variety } \\
\hline & \multicolumn{2}{|c|}{ Da Hua aromatic glutinous } & \multicolumn{2}{|c|}{ Hong Shi I } & \multicolumn{2}{|c|}{ Tian You VIII } & \multicolumn{2}{|c|}{ Xin Dao X } & \multicolumn{2}{|c|}{ Yu Jing VI } \\
\hline & Mean & SD & Mean & SD & Mean & SD & Mean & SD & Mean & SD \\
\hline Length $/ \mathrm{mm}$ & 7.03 & 0.11 & 8.02 & 0.06 & 7.54 & 0.07 & 7.13 & 0.08 & 7.46 & 0.13 \\
\hline Width/mm & 3.74 & 0.06 & 3.32 & 0.08 & 3.45 & 0.05 & 3.13 & 0.07 & 3.27 & 0.10 \\
\hline Thickness/mm & 1.88 & 0.04 & 2.42 & 0.07 & 2.19 & 0.04 & 2.28 & 0.06 & 2.29 & 0.08 \\
\hline Volume $/ \mathrm{mm}^{2}$ & 18.64 & 0.57 & 18.21 & 0.10 & 17.04 & 0.19 & 16.57 & 0.11 & 17.18 & 0.16 \\
\hline Surface area $/ \mathrm{mm}^{2}$ & 19.25 & 0.24 & 18.46 & 0.32 & 17.10 & 0.11 & 16.37 & 0.10 & 16.82 & 0.25 \\
\hline Needle degree & 3.84 & 0.09 & 3.97 & 0.06 & 3.19 & 0.38 & 3.53 & 0.05 & 3.21 & 0.30 \\
\hline Flat degree & 1.68 & 0.04 & 1.61 & 0.05 & 1.57 & 0.05 & 1.51 & 0.06 & 1.49 & 0.04 \\
\hline Shape factor & 2.28 & 0.08 & 2.47 & 0.09 & 2.04 & 0.25 & 2.34 & 0.10 & 2.15 & 0.21 \\
\hline Sphericity & 1.44 & 0.09 & 1.43 & 0.03 & 1.35 & 0.03 & 1.33 & 0.02 & 1.40 & 0.03 \\
\hline
\end{tabular}

Note: SD is the standard deviation.

In Figure 8, A denotes the results for the input vector consisted of nine 3D morphological features; $\mathrm{B}$ denotes the results for the input vector consisted of nine projection features of the main cross-section plane of rice seeds; $\mathrm{C}$ denotes results for the input vector consisted of all 18 features.

The results of our method were compared with the results of methods of Yan Qian, Sakai, and Zhijun Wang. The comparison results are presented in Table 3 and explained in the following.

Qian et al. ${ }^{[20]}$ managed to increase the recognition rate of rice seeds up to $90 \%$ by inputting eight measured three-dimensional rice seeds data into a BP neural network. However, in our experiments, nine 3D morphological features of rice seeds, which could be obtained by a 3D line laser scanner, were used as an input vector with higher accuracy, so higher recognition rate was achieved.

Sakai et al. ${ }^{[21]}$ analyzed the shape of brown rice seeds and polished rice seeds, and used an image processing technology to extract the cross-section area, perimeter, major axis length, minor axis length, aspect ratio, and circularity of rice seeds, and to classified them. The results showed that the classification accuracy of $95.4 \%$ could be achieved by classifying the brown rice seeds and polished rice seeds by the shape features. Therefore, it was still not enough to use only the projection features of the main plane cross-section of rice seeds with the average recognition rate of $92.6 \%$ as an input vector in rice seeds identification.

Wang et al. ${ }^{[22]}$ extracted 24 wheat image features with an average recognition rate of $93 \%$ by using the input vector consisted of all the features, including area, perimeter, equivalent circle diameter, the maximum and minimum radius, elliptic eccentricity, circularity, ovality, squareness, and so on.

Compared with the several above-mentioned studies, our method not only increased the identification accuracy of rice varieties but also improved the recognition rate, as shown in Table 3. Besides, for the same rice seed species, the highest recognition rate was achieved when all 18 feature vectors were used as an input of the BP network.

Table 3 Comparison results

\begin{tabular}{lcccc}
\hline \multirow{2}{*}{$\begin{array}{c}\text { Comparison } \\
\text { parameter }\end{array}$} & Qian $^{[20]}$ & Sakai $^{[21]}$ & Wang $^{[22]}$ & Our method \\
\cline { 2 - 5 } & rice seeds & rice seeds & wheat seeds & rice seeds \\
\hline Identification Object & 8 & 6 & 24 & 18 \\
Feature number & $90 \%$ & $95.4 \%$ & $93 \%$ & $97 \%$ \\
Recognition rate & & & &
\end{tabular}

\section{Conclusions}

In this paper, the classification of rice seeds is studied. Following the characteristics of rice varieties, laser scanning technology is used to measure the morphological characteristics of rice seeds. Based on the noise filtering, smoothing of the point cloud, vulnerability repair, and downsampling reduction, and by using the morphological characteristics of rice seeds, a method for calculation of rice seed morphological characteristics is proposed. Using the proposed method, the three-dimensional morphological characteristics of a rice seed surface and the projection features of the main plane cross-section were obtained. The rice seed 
recognition was conducted by the BP neural network model using respectively three different input vectors containing: (1) nine three-dimensional morphological characteristics of rice seeds, (2) nine projection features of the main plane of rice seeds, and (3) all 18 features. The results of the tests conducted with five rice seed varieties showed that the recognition rate was the highest when the input vector consisted of all 18 features and the recognition rates of the five rice seed varieties were $96 \%, 97 \%$, $91 \%, 94 \%$, and $90 \%$, and the average recognition rate was $94 \%$.

Although the presented method was proven to have extremely good performance, some of the techniques used in this study are in the developing phase but not mature yet, such as the point cloud of rice seeds; so, further research is needed, which will be the subject of our future work.

\section{Acknowledgements}

The authors are very grateful for the support provided by the $\mathrm{N}$ ational Natural Science Foundation of China (Grant No. 51507081) , the Fundamental Research Funds for the Central Universities (KJ QN201623), and the National Key Research and Development Pro gram of China (2017YFD0700800).

\section{[References]}

[1] Vu H, Tachtatzis C, Murray P, Harle D, Dao T K, Atkinson R, et al Spatial and spectral features utilization on a Hyperspectral imaging system for rice seeds varietal purity inspection. 12th IEEE-RIVF Internationa Conference on Computing \& Communication Technologies, Hanoi, Vietnam, 2016.

[2] Li J F, Chen M, Jin X B, Chen Y, Dai Z Y, Ou Z H, et al. Calibration of a multiple axes 3-D laser scanning system consisting of robot, portable laser scanner and turntable. Optik - International Journal for Light and Electron Optics, 2011; 122(4): 324-329.

[3] Dai K K, Zhao J W, Cao F L. A novel decorrelated neural network ensemble algorithm for face recognition. Knowledge-Based Systems, 2015; 89: 541-552.

[4] Lu J, Zhao J W, Cao F L. Extended feed forward neural networks with random weights for face recognition. Neurocomputing, 2014; 136(1): 96-102.

[5] Li H M, Zhang R J. Research of vehicle license plate recognition based on BP neural network. Computer Engineering \& Design, 2010; 31(3): 619-621.

[6] Chen Z, Huang L L. Palmprint recognition based on ICA and BP neural network. Journal of Beijing University of Aeronautics \& Astronautics, 2008; 34(3): 290-293

[7] Zhang J H, Qi L J, Ji R H, Wang H, Huang S K, Wang P. Cotton diseases identification based on rough sets and BP neural network. Transactions of the CSAE, 2012; 28(7): 161-167. (in Chinese)

[8] Wu J, Huang F R, Huang C H, Zhang J, Chen X D. Study on near infrared spectroscopy of transgenic soybean identification based on principal component analysis and neural network. Spectroscopy and Spectral Analysis, 2013; 33(6): 1537-1541.

[9] Cao W S, Zhang C Q, Wang J X, Liu S X, Xu X Z. Purity identification of maize seed based on discrete wavelet transform and BP neural network. Transactions of the CSAE, 2012; 28: 253-258. (in Chinese)

[10] Tao Y T, Zhou J. An Automatic Segmentation and Recognition Method of Apple Tree Point clouds in the Real Scene Based on the Fusion of Color and 3D Feature. 2017 ASABE Annual International Meeting, Spokane, USA, 2017

[11] Shantaiya S, Ansari U. Identification of food grains and its quality using pattern classification. IEEE International Conference on Communication Technology, Raipur, India, 2010

[12] Li H, Qian Y, Cao P, Yin W Q, Dai F, Hu F, Yan Z J. Calculation method of surface shape feature of rice seeds based on point cloud. Computers \& Electronics in Agriculture, 2017; 142: 416-423.

[13] Yao L, Dong G S, Tang H T, Long W Z. Application to Body Surface Burn Area Estimation Using 3D Imagining Techniques. Journal of Donghua University(Natural Science), 2015; 41(1): 84-90.

[14] Gao Y H M, Zheng J Z, Chen J. Method of Calculating Forest Land Surface Area based on Automatic Boundary Extraction. Modern Surveying and Mapping, 2015; 2: 19-22.

[15] Marton Z C, Rusu R B, Beetz M. On fast surface reconstruction methods for large and noisy point clouds. IEEE International Conference on Robotics and Automation, Kobe, Japan, 2009.

[16] Liu K. Coarse Aggregate Shape characteristics Analysis Based on Digital Image Technology. Guangdong Highway Communications, 2011; 4: $1-6$.

[17] Chen Z, Ma H C, Zhang L. Cloverleaf interchange boundary extraction from airborne LiDAR data based on advanced neighborhood structure and contour analysis. Journal of Remote Sensing, 2013; 17(5): 1146-1157.

[18] Mo C X, Wei W, Xia B L, Wang D B, Sun G K, Liu L, Jiang Q L. Application of cubic spline interpolation function in flood routing. Water Power, 2013; 39(10): 12-14.

[19] Petrosian A R, Gurzadyan V G, Hendry M A, Nikoghossian E H. One arc degree core substructure of the virgo cluster. Astrophysics, 1998; 41(1): 32-40.

[20] Qian Y, Yin W Q, Lin X Z, Ding Y Q, Feng X B. Variety identification of rice seed based on three-dimensional reconstruction method of sequence images. Transactions of the CSAE, 2014; 30(7): 190-196. (in Chinese)

[21] Sakai N, Yonekawa S, Matsuzaki A, Morishima H. Two-dimensional image analysis of the shape of rice and its application to separating varieties. Journal of Food Engineering, 1996; 27(4): 397-407.

[22] Wang Z J, Cong P S, Zhou J L, Zhu Z L. Method for identification of external quality of wheat grain based on image processing and artificial neural network. Transactions of the CSAE, 2007; 23(1): 158-161. (in Chinese) 